\title{
Imaging and Quantification of the Area of Fast-Moving Microbubbles Using a High-Speed Camera and Image Analysis
}

\author{
Nina Vyas ${ }^{1}$, Mehdi Mahmud ${ }^{2}$, Qianxi X Wang $^{2}$, A. Damien Walmsley ${ }^{1}$ \\ ${ }^{1}$ School of Dentistry, University of Birmingham ${ }^{2}$ Department of Mathematics, University of Birmingham
}

\section{Corresponding Authors}

Nina Vyas

N.Vyas@bham.ac.uk

A. Damien Walmsley

a.d.walmsley@bham.ac.uk

\section{Citation}

Vyas, N., Mahmud, M., Wang, Q.X.,

Walmsley, A.D. Imaging and

Quantification of the Area of Fast-Moving

Microbubbles Using a High-Speed

Camera and Image Analysis. J. Vis.

Exp. (163), e61509, doi:10.3791/61509

(2020).

\section{Date Published}

September 5, 2020

DOI

$10.3791 / 61509$

\section{URL}

jove.com/video/61509

\section{Abstract}

An experimental and image analysis technique is presented for imaging cavitation bubbles and calculating their area. The high-speed imaging experimental technique and image analysis protocol presented here can also be applied for imaging microscopic bubbles in other fields of research; therefore, it has a wide range of applications. We apply this to image cavitation around dental ultrasonic scalers. It is important to image cavitation to characterize it and to understand how it can be exploited for various applications. Cavitation occurring around dental ultrasonic scalers can be used as a novel method of dental plaque removal, which would be more effective and cause less damage than current periodontal therapy techniques. We present a method for imaging the cavitation bubble clouds occurring around dental ultrasonic scaler tips using a high-speed camera and a zoom lens. We also calculate the area of cavitation using machine learning image analysis. Open source software is used for image analysis. The image analysis presented is easy to replicate, does not require programming experience, and can be modified easily to suit the application of the user.

\section{Introduction}

Imaging the motion of bubbles is important for various applications because it controls the hydrodynamics of a system. There are many applications where this can be useful: in fluidized bed reactors ${ }^{1,2}$, or for cleaning with cavitation bubbles $^{3,4}$. The purpose of imaging bubbles is to understand more about the bubble dynamics or about the direction and motion of a cloud of bubbles. This can be done through observing structures imaged and also by using image analysis to obtain quantitative information, such as the size of the bubbles.

Cavitation bubbles are gas or vapor entities that occur in a fluid when the pressure drops below the saturated pressure value $^{5}$. They can occur when an acoustic field is applied 
to a fluid at ultrasonic frequencies. They repeatedly grow and collapse, and upon collapse can release energy in the form of high-speed micro-jets and shockwaves ${ }^{6,7}$. These can dislodge particles on a surface through shear forces and cause surface cleaning ${ }^{8}$. Cavitation bubbles are being investigated for surface cleaning in different industries, such as for semiconductors, food, and wound cleaning $9,10,11,12$. They could also be used to clean dental plaque from teeth and biomaterials such as dental implants ${ }^{12,13}$. Cavitation occurs around currently used dental instruments such as ultrasonic scalers and endodontic files and shows potential as an additional cleaning process with these instruments ${ }^{14}$.

The oscillation of cavitation bubbles occurs over a few microseconds and therefore a high-speed camera is required to capture their motion by imaging at thousands of frames per second ${ }^{8}$. We demonstrate a method of imaging microbubble cavitation around dental ultrasonic scalers. The aim is to understand how cavitation varies around different ultrasonic scalers, so it can be optimised as a novel way to clean dental plaque.

Previous methods used to investigate the cavitation include sonochemiluminesence, which uses luminol to detect where cavitation has occurred ${ }^{15,16}$. However, this is an indirect technique and it is not able to visualize the cavitation bubbles in real time. Therefore, it is not able to accurately determine exactly where it happens on the instrument, and no information can be gained on the bubble dynamics, unless it is combined with other imaging techniques ${ }^{17}$. Highspeed imaging can image not only the cavitation bubbles growing and collapsing but also the type of cavitation occurring: cavitation clouds, microstreamers and microjets $^{6,7,18}$. These give more information about how the cavitation can clean surfaces.
We present a method of imaging cavitation microbubbles using a high-speed camera and calculating the mean area of cavitation occurring. This method is demonstrated using an example of cavitation occurring around different dental ultrasonic scaler tips, although the experimental and image analysis steps can be used for other applications, such as for imaging other macro and microbubbles.

\section{Protocol}

\section{Instrument setup}

1. Select the instrument or object to be imaged. In this experiment an ultrasonic scaler was imaged. Cavitation bubbles occur around the tips of ultrasonic scalers in water.

2. Select a micro positioning stage for the instrument to be imaged with $X Y Z$ translation and rotation. Place on a laboratory jack. Attach the instrument handle to the micro positioning stage

3. Select an optically transparent water container for imaging. The container used in these experiments was created with glass microscope slides.

4. Select an $X Y$ stage with a rotation platform. Place on a laboratory jack. Place the water container on the stage and fill with filtered water (reverse osmosis or distilled).

\section{High-speed camera setup}

1. Select a high-speed camera with the desired frame rate and resolution and a high intensity light source with a fibre light guide.

2. Attach a micropositioning sliding plate to the high-speed camera body and connect it to a tripod stand. 
3. Select a lens with the desired resolution and focal length and attach this to the camera. For this experiment a zoom lens was used at a resolution of $8.4 \mu \mathrm{m} / \mathrm{pixel}$.

4. Fill the imaging tank with water and position the tip of the instrument to be imaged in the water tank in the desired orientation.

5. After connecting the camera and loading the live view in the software, use low magnification to focus on the tip of the ultrasonic scaler, repositioning the light source if necessary. Position the instrument and the light source in front of the camera and focus. Adjust to the desired frame rate and brightness.

NOTE: A higher light intensity is required for imaging at high frames rates, short shutter speeds and/or high magnifications. Illumination can be provided in reflection mode or transmission mode. In this protocol the illumination is provided in transmission mode (bright field) using a high intensity cold illumination device.

6. Set an optimal frame rate and shutter speed for the highspeed camera. In this experiment the frame rate was 6400 fps with a shutter speed of 262 nanoseconds. A short shutter speed is required for fast moving bubbles such as cavitation bubbles to ensure that they are in focus.

7. Adjust the magnification of the zoom lens and the intensity of the light source so the background is white without being overexposed.

\section{Calibration}

1. Record the position of the tip (rotation in $x$-y stage, rotation angle of instrument for reproducibility).

2. To ensure the field of view is consistent for each repeat, choose a reference point and note down the coordinates. In this case the reference point was the tip of the ultrasonic scaler. It can then be repositioned in future experiments in the same place within the field of view.

3. If the pixel size is unknown, image a graticule with $10 \mu \mathrm{m}$ markings at the set magnification and use image analysis software such as Fiji to calculate the resolution.

\section{High-speed video recording}

1. Image the instrument without cavitation. This will be subtracted from the cavitation images in image analysis when calculating the area of the cavitation bubbles. Save the videos in a format such as TIFF so no image quality is lost.

2. Image the instrument operating with cavitation. Ensure there are sufficient frames for accurate analysis, for example 5 repeats with 500 frames each.

\section{Image processing}

1. Download $\mathrm{Fiji}^{19}$ from the ImageJ website (https:// imagej.net/Fiji). An ImageJ macro code has been provided which automatically does the image analysis steps described below and can also be changed to suit the application. The individual steps of the macro are described in steps 5.3-5.5.

2. Crop the image to remove any darker areas resulting from uneven illumination, if needed. Ensure that all images are cropped to the same size and at the identical point in the image.

3. Convert the images to binary by automatically thresholding using one of the auto thresholds. In this example the minimum auto threshold is used.

4. Run the fill holes command to remove any black pixels from inside the bubbles which were falsely segmented. 
5. Calculate the histogram of the stack to show the number of pixels corresponding to the scaler and the cavitation in each frame.

6. In this case the pixels corresponding to the bubbles are white and have value 255 . Save these measurements.

7. Repeat steps 5.3-5.6 for the video of the instrument operating without the bubbles.

8. Calculate the mean area of the ultrasonic scaler tip only from the results of the histogram.

9. Subtract the mean area of the instrument from each of the areas calculated from the videos of the bubbles around the scaler. The area of the bubbles is left to measure.

10. Visualize by subtracting the binary image of the scaler from the binary image of the scaler with bubbles using the image calculator in Fiji.

11. Calculate the mean and standard deviation of the area of the bubbles.

12. Convert the values from number of pixels to area (in this case $\mu \mathrm{m}^{2}$ ) by multiplying by the pixel size squared. Calculate the size of each pixel by imaging a graticule with the high-speed camera at the same magnification as was used for imaging and use ImageJ to set the scale.

13. Plot the data. It is also possible to conduct statistical analysis to show any significant difference in the area of bubbles if comparing different conditions.

\section{ImageJ macro}

1. In the ImageJ/Fiji menu, go to Plugins $>$ New $>$ Macro. Ensure IJ1 Macro is checked under the language menu and copy and paste the following code. Click run to execute the macro (Supplementary File).

\section{Representative Results}

The image analysis steps can be seen in Figure 1 for one of the ultrasonic scaler tips tested. A FSI 1000 tip and a 10P tip were imaged inside a water tank with the cooling water turned off (Figure 2). Cavitation occurred near the bend of tip FSI 1000 at maximum power, and near the free end in tip 10P (Figure 3 and Figure 4). The mean area of cavitation was 0.1 $\pm 0.07 \mathrm{~mm}^{2}$ for the FSI 1000 tip and $0.50 \pm 0.25 \mathrm{~mm}^{2}$ for the 10P tip (Figure 5). 

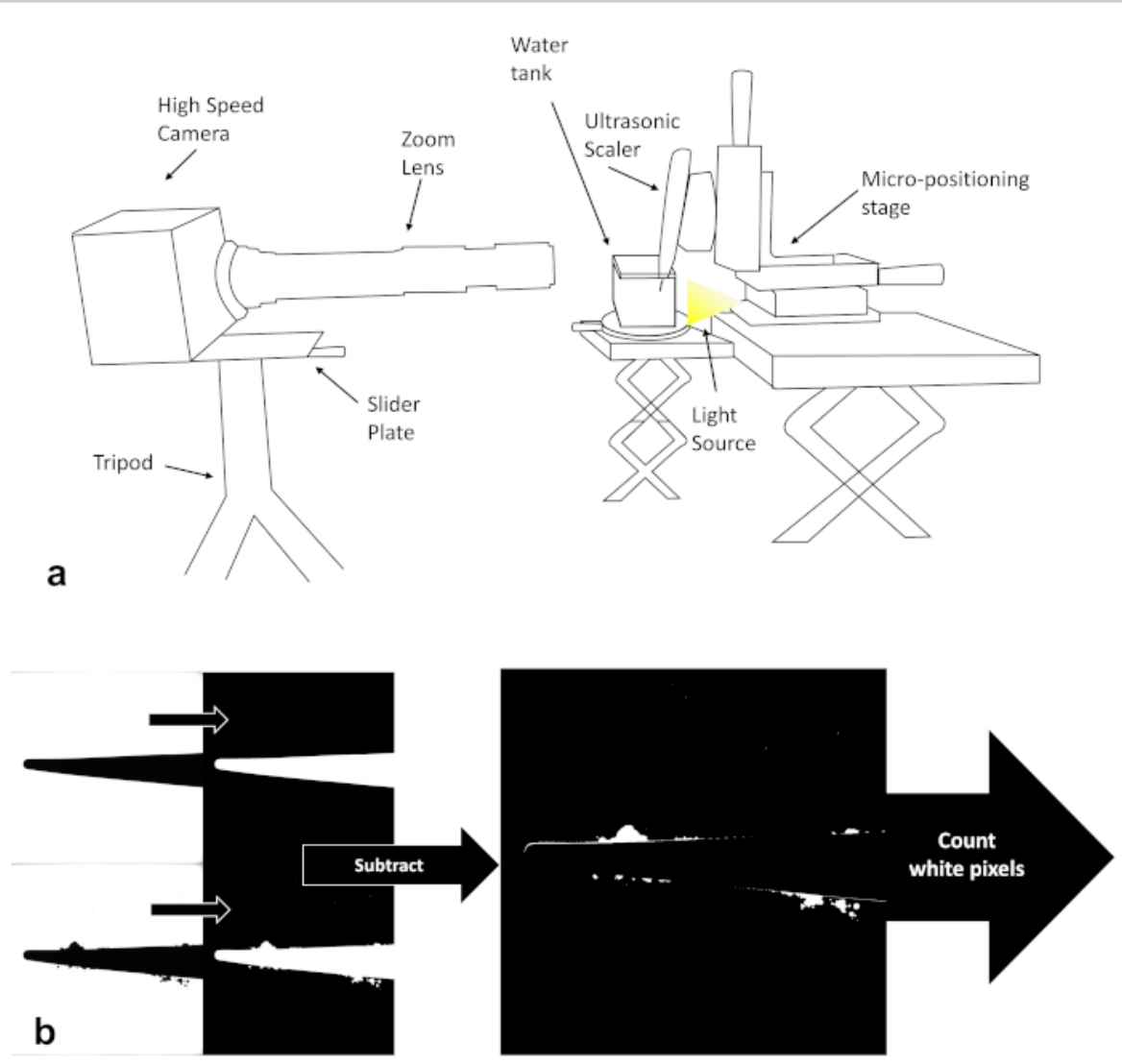

Figure 1: High-speed imaging setup and image analysis steps (a) Schematic of the high-speed imaging setup used in the study. (b) Schematic of the image analysis steps used in the study, showing the raw images on the left of the scaler tip only and with cavitation, which were then binarized and subtracted from each other to calculate the area of the cavitation clouds. Please click here to view a larger version of this figure. 


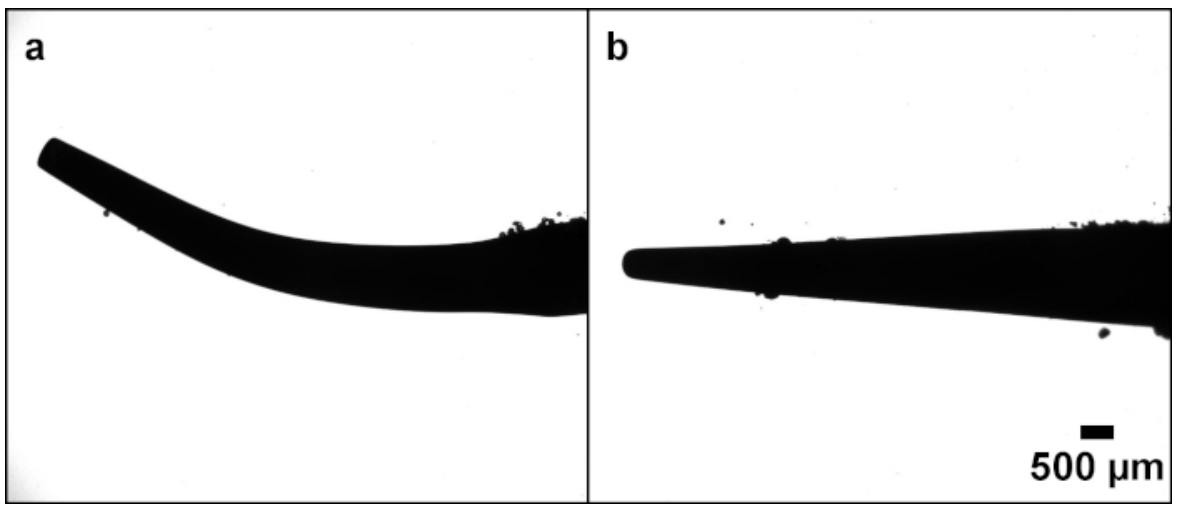

Figure 2: Comparison between different tips High-speed image stills showing cavitation occurring around the two ultrasonic scaler tips tested (a) FSI 1000 (b) 10P. Please click here to view a larger version of this figure.

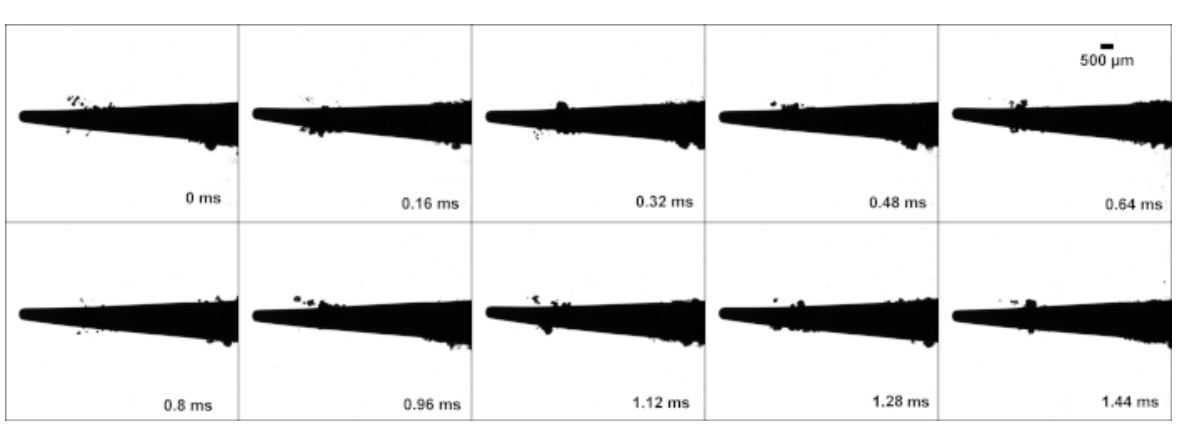

Figure 3: Tip 10P high-speed images: High-speed image stills of tip 10P, from a video taken at 6400 frames per second. Cavitation can be seen around the free end of the tip. Please click here to view a larger version of this figure. 


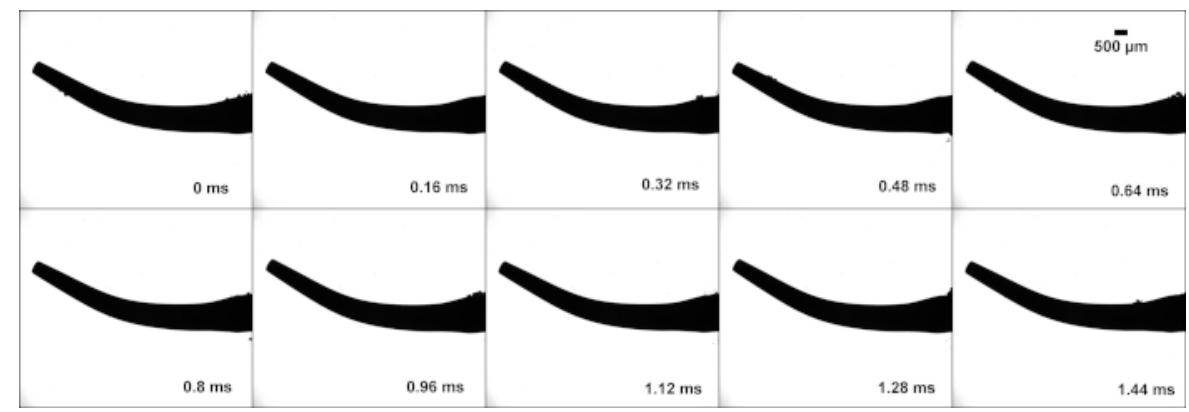

Figure 4: Tip FSI1000 high-speed images: High-speed image stills of tip FSI 1000, from a video taken at 6400 frames per second. Cavitation can be seen around the middle of the tip. Please click here to view a larger version of this figure.

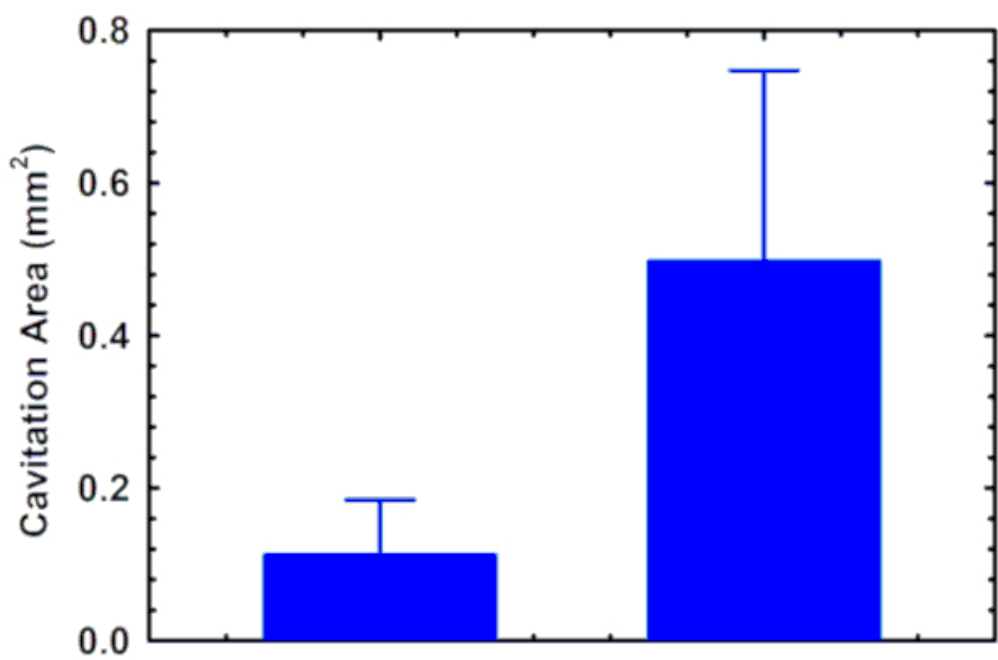

Tip FSI-1000

Tip 10P

Figure 5: Cavitation area image analysis results. The mean area of cavitation occurring around the FSI 1000 and $10 \mathrm{P}$ ultrasonic scaler tips calculated using the image analysis technique described. The error bars represent the standard deviation. Please click here to view a larger version of this figure.

Supplementary File. Please click here to download this file.

\section{Discussion}

The technique described in this paper enables imaging of fast-moving microbubbles with high spatial and temporal resolution. It can potentially benefit a wide range of scientific disciplines such as chemical engineering, dentistry and medicine. Engineering applications include imaging cavitation bubbles for cleaning surfaces, or for imaging bubbles in fluidized bed reactors. Biomedical applications include imaging cavitation around medical and dental instruments 
and imaging biofilm debridement from hard and soft tissue using cavitation bubbles. In this study we demonstrated the technique by imaging cavitation around two different dental ultrasonic scaler tips. The amount of cavitation varies between the two tips tested in this study, with more cavitation clouds observed around the free end of tip 10P. This has previously been linked to vibration amplitude ${ }^{20}$. The highspeed videos show that the FSI 1000 tip has less vibration, which is likely to be why there is less cavitation around this tip.

One limitation of the image analysis method is that the image subtraction technique to remove the area of the scaler is not completely accurate because the scaler is oscillating and therefore the subtraction may leave some areas of the scaler falsely segmented as bubbles. However, this has been accounted for by averaging the area from a large number of frames $(n=2000)$. This would not be a problem for applications where the object to be subtracted is stationary. For studies where the moving object to be subtracted has a much higher variance, we recommend synchronizing the movements in both videos before subtracting for accurate results. In the current study, we did not synchronize the oscillations but since the vibration was low, we can assume that the oscillations correspond well to each other in these two measurements.

The image thresholding is accurate because the brightfield illumination provides a uniform background with good contrast. It is critical to ensure that the background is uniform and does not contain any other objects which could be falsely segmented. The thresholding method can be modified by using other automatic thresholds to suit the application. Manual thresholding, where the user sets the threshold value, is also possible but is not recommended as it reduces the reproducibility of the results, since different users will select different threshold values.

Image analysis has been used for many other bubble imaging studies. These also use a similar method of backlighting to get optimum contrast between the bubbles and the background, and thresholding to segment the bubbles $21,22,23,24$. The method shown in the current study can also be generalized to be used for many different bubble imaging applications, which are not limited to only high-speed imaging. High-speed imaging has been used for cavitation bubbles generated in water and also around instruments such as endodontic files and ultrasonic scalers $12,25,26,27,28$. For example Rivas et al. and Macedo et al. used a high-speed camera attached to a microscope, with illumination provided by a cold light source to image cleaning with cavitation, and to image cavitation around an endodontic file ${ }^{17,29}$. Bright field illumination provides more contrast between the background and the bubbles, making it possible to use simple segmentation techniques such as thresholding, as demonstrated by Rivas et al. for imaging and quantifying cavitation erosion and cleaning over time ${ }^{29}$. Dark field illumination makes thresholding more difficult due to the higher variation in grey scales $^{4,30}$. Image analysis has been used in other studies to gather more information about bubbles ${ }^{1,2}$. Vyas et al. used a machine learning approach to segment cavitation bubbles around an ultrasonic scaler ${ }^{20}$. The method described in the current paper is quicker because it uses simple thresholding so it is less computationally intensive, and bubbles occurring above and below the scaler can be analysed. However, the thresholding method used in the current paper is only accurate if the background is uniform. If it is not possible to obtain a uniform background during imaging, other image processing techniques can be used such as the use of background subtraction using a rolling ball radius to correct 
for uneven illumination, filtering using median or Gaussian filters to remove noise, or also using machine learning based techniques ${ }^{20,31}$.

In conclusion, we present a high-speed imaging and analysis protocol to image and calculate the area of a microscopic moving object. We have demonstrated this method by imaging cavitation bubbles around an ultrasonic scaler. It can be used for imaging cavitation around other dental instruments such as endodontic files and it can be easily adapted for other non-dental bubble imaging applications.

\section{Disclosures}

The authors have nothing to disclose.

\section{Acknowledgments}

The authors are grateful for funding from the Engineering and Physical Sciences Research Council EP/P015743/1.

\section{References}

1. Asegehegn, T. W., Schreiber, M., Krautz, H. J. Investigation of bubble behavior in fluidized beds with and without immersed horizontal tubes using a digital image analysis technique. Journal of Power Technologies. 210 (3), 248-260 (2011).

2. Busciglio, A., Vella, G., Micale, G., Rizzuti, L. Analysis of the bubbling behaviour of 2D gas solid fluidized beds: Part I. Digital image analysis technique. Chemical Engineering Journal. 140 (1), 398-413 (2008).

3. Versluis, M. High-speed imaging in fluids. Experiments in Fluids. 54 (2), 1-35 (2013).

4. Matsumoto, H., Yoshimine, Y., Akamine, A. Visualization of irrigant flow and cavitation induced by Er: YAG laser within a root canal model. Journal of Endodontics. 37 (6), 839-843 (2011).

5. Young, F. R. Cavitation. World Scientific, (1999).

6. Brennen, C. E. Cavitation and Bubble Dynamics. Cambridge University Press, (2013).

7. Leighton, T. The acoustic bubble. Academic Press, (2012).

8. Verhaagen, B., Rivas, D. F. Measuring cavitation and its cleaning effect. Ultrasonics Sonochemistry. 29, 619-628 (2016).

9. Oulahal-Lagsir, N., Martial-Gros, A., Boistier, E., Blum, L., Bonneau, M. The development of an ultrasonic apparatus for the non-invasive and repeatable removal of fouling in food processing equipment. Letters in Applied Microbiology. 30 (1), 47-52 (2000).

10. Gale, G. W., Busnaina, A. A. Roles of cavitation and acoustic streaming in megasonic cleaning. Particulate Science and Technology. 17 (3), 229-238 (1999).

11. Erriu, M. et al. Microbial biofilm modulation by ultrasound: Current concepts and controversies. Ultrasonics Sonochemistry. 21 15-22 (2014).

12. Van der Sluis, L., Versluis, M., Wu, M., Wesselink, P. Passive ultrasonic irrigation of the root canal: a review of the literature. International Endodontic Journal. 40 (6), 415-426 (2007).

13. Vyas, N., Sammons, R. L., Addison, O., Dehghani, H., Walmsley, A. D. A quantitative method to measure biofilm removal efficiency from complex biomaterial surfaces using SEM and image analysis. Scientific Reports. 6, 32694 (2016).

14. Walmsley, A. D., Lea, S. C., Felver, B., King, D. C., Price, G. J. Mapping cavitation activity around dental ultrasonic 
tips. Clinical Oral Investigations. 17 (4), 1227-1234 (2013).

15. Price, G. J., Tiong, T. J., King, D. C. Sonochemical characterisation of ultrasonic dental descalers. Ultrasonics Sonochemistry. 21, 2052-2060 (2014).

16. Felver, B., King, D. C., Lea, S. C., Price, G. J., Damien Walmsley, A. Cavitation occurrence around ultrasonic dental scalers. Ultrasonics Sonochemistry. 16, 692-697 (2009).

17. Macedo, R. G. et al. Sonochemical and high-speed optical characterization of cavitation generated by an ultrasonically oscillating dental file in root canal models. Ultrasonics Sonochemistry. 21, 324-335 (2014).

18. Reuter, F., Lauterborn, S., Mettin, R., Lauterborn, W. Membrane cleaning with ultrasonically driven bubbles. Ultrasonics Sonochemistry. 37, 542-560 (2017).

19. Schindelin, J. et al. Fiji: an open-source platform for biological-image analysis. Nature Methods. 9 (7), 676-682 (2012).

20. Vyas, N. et al. High-speed Imaging of Cavitation around Dental Ultrasonic Scaler Tips. PLoS One. 11 (3), e0149804 (2016).

21. Ahmed, F. S., Sensenich, B. A., Gheni, S. A., Znerdstrovic, D., Al Dahhan, M. H. Bubble dynamics in 2D bubble column: comparison between high-speed camera imaging analysis and 4-point optical probe. Chemical Engineering Communications. 202 (1), 85-95 (2015).

22. Honkanen, M. Reconstruction of three-dimensional bubble surface from high-speed orthogonal imaging of dilute bubbly flow. Proceedings of Computational Methods in Multiphase Flow V, New Forest, UK. 469-480 (2009).
23. do Amaral, C. E. et al. Image processing techniques for high-speed videometry in horizontal two-phase slug flows. Flow Measurement and Instrumentation. 33, 257-264 (2013).

24. Lau, Y., Deen, N., Kuipers, J. Development of an image measurement technique for size distribution in dense bubbly flows. Chemical Engineering Science. 94, 20-29 (2013).

25. Matsumoto, Y., Yoshizawa, S. Behaviour of a bubble cluster in an ultrasound field. International Journal for Numerical Methods in Fluids. 47 (6-7), 591-601 (2005).

26. Peeters, H. H., Iskandar, B., Suardita, K., Suharto, D. Visualization of removal of trapped air from the apical region of the straight root canal models generating 2-phase intermittent counter flow during ultrasonically activated irrigation. Journal of Endodontics. 40 (6), 857-861 (2014).

27. Halford, A. et al. Synergistic effect of microbubble emulsion and sonic or ultrasonic agitation on endodontic biofilm in vitro. Journal of Endodontics. 38 (11), 1530-1534 (2012).

28. Kauer, M., Belova-Magri, V., Cairós, C., Linka, G., Mettin, R. High-speed imaging of ultrasound driven cavitation bubbles in blind and through holes. Ultrasonics Sonochemistry. 48, 39-50 (2018).

29. Rivas, D. F. et al. Localized removal of layers of metal, polymer, or biomaterial by ultrasound cavitation bubbles. Biomicrofluidics. 6 (3), 034114 (2012).

30. Pishchalnikov, Y. A. et al. Cavitation Bubble Cluster Activity in the Breakage of Kidney Stones by Lithotripter Shockwaves. Journal of Endourology. 17 (7), 435-446 (2003). 
31. Sternberg, S. R. Biomedical image processing.

Computer. (1), 22-34 (1983). 